\title{
Features of a Cycloid Speed Increaser with Double Satellite Gear for Small Mechatronic Wind and Hydro Systems
}

\author{
C. Jaliu ${ }^{1}$, D.V. Diaconescu ${ }^{1}$, M. Neagoe ${ }^{1}$ and R. Săulescu ${ }^{1}$ \\ ${ }^{1}$ Product Design and Robotics Department, Transilvania University of Brasov \\ no. 29, Eroilor Boulevard, 500036, Brasov, Romania, Phone/Fax number:+40 268 472496, \\ cjaliu@unitbv.ro,dvdiaconescu@unitbv.ro,mneagoe@unitbv.ro, rsaulescu@unitbv.ro.
}

\begin{abstract}
The paper main objective is to extend the base of speed increasers to be used in renewable energy systems, taking into account requirements regarding the multiplication ratio, efficiency and overall dimension. In order to fulfil this objective, a cycloid speed reducer produced by Onvio Company is considered and analyzed as speed increaser, for which the synthesis of the teeth numbers is made. Then, a comparative analysis of the kinematical and dynamic transmission features of the Onvio type transmission used both as speed reducer and speed increaser is presented in the paper.
\end{abstract}

\section{Key words}

Onvio type reducer, cycloid reducer, cycloid speed increaser, multiplication ratio, efficiency.

\section{Introduction}

Usually, the mountain area is characterized by low water heads and small and medium discharges, as well as medium wind power; therefore, in order to generate a sufficient energy to fulfil the needs of isolated consumers or of small communities living in these geographical conditions, it is necessary to multiply the energy produced by the turbine by using a speed increaser between the turbine and the generator (a water turbine connected directly to the generator, that can produce the needed energy only for higher values of the discharges is presented in Fig. 1). From economical reasons, the speed increasers have to be simple, reliable, with a higher efficiency, a multiplication ratio between 5 and 30 and an easier maintenance. There are several options for coupling in wind and hydro systems that allow an increase in speed: belts (hydro), chains (hydro) and gears (wind and hydro) (Fig. 2). Each has its advantages and disadvantages [2].

A source that can be used in developing new schemes of speed increasers for wind turbines and hydro stations is represented by the planetary reducers. Some representative solutions of 1 DOF simple planetary increasers developed from reducers with a large technical use, by inverting the energy flow were presented and analyzed comparatively in the previous papers $[2,3]$.

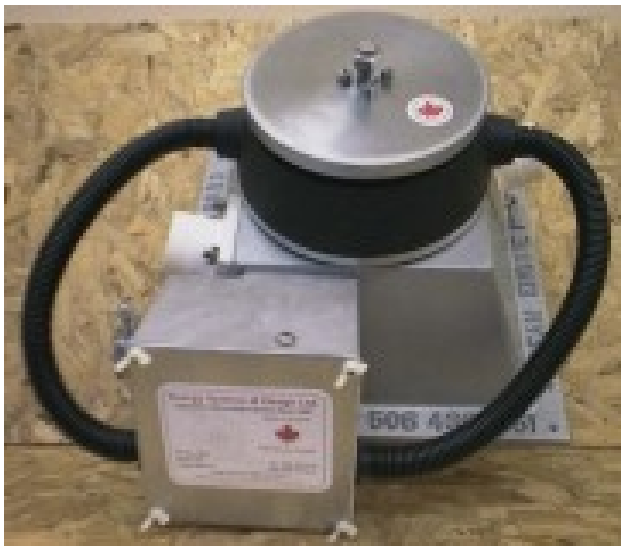

Fig. 1. Turgo water turbine connected directly to the generator

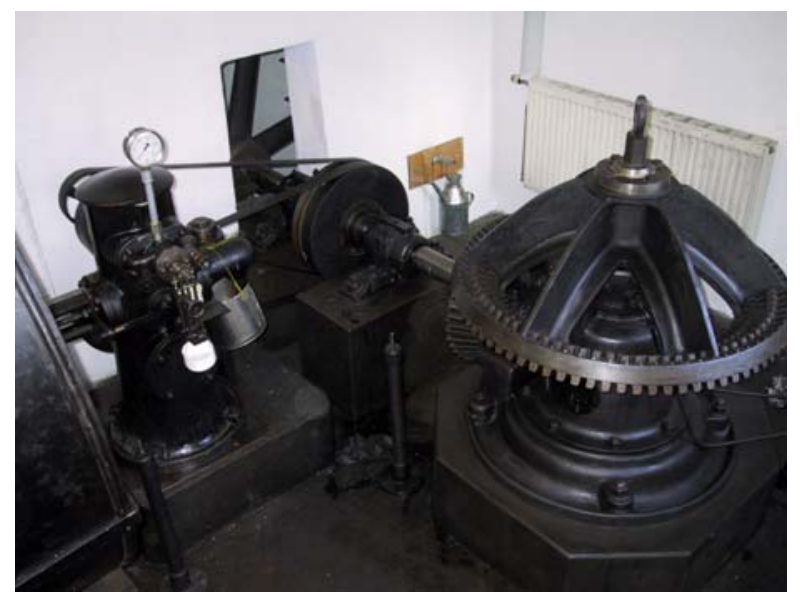

Fig. 2. Bevel gear pair used as speed increaser in a small hydro power station

Thus, in order to extend the library of speed increasers, solutions of cycloid reducers with simple satellite gears were analyzed as speed increasers [3].

This paper analyzes the possibility of using as speed increaser, fulfilling also the previous requirements, the cycloid reducers with double satellite gears of Onvio type (like those produced by Onvio Company, see Fig. $3, a)$. 


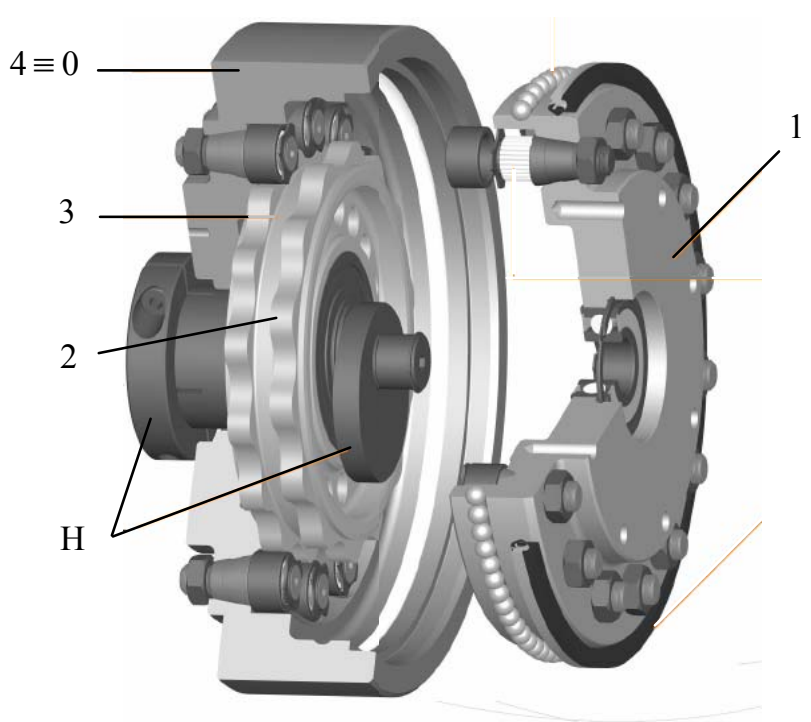

a)

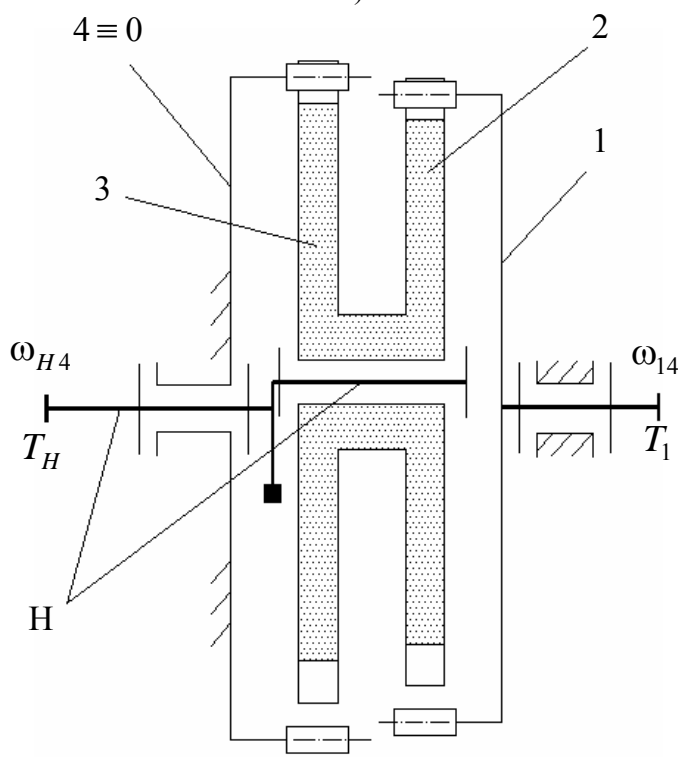

b)

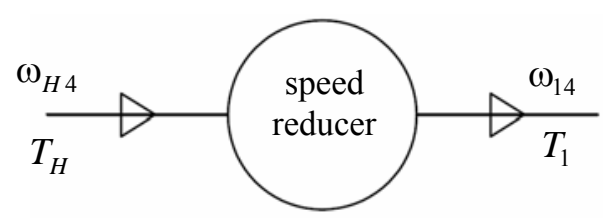

c)

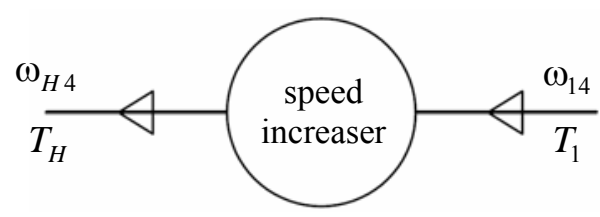

d)

Fig. 3. a) Cycloid reducer of Onvio type, b) structural diagram and block diagram of the transmission in both cases: c) speed reducer and d) speed increaser.

In order to analyze the functioning as speed increaser, the 1 DOF Onvio type transmission will be mounted between the turbine and the generator in a Turgo existing turbine (see Fig.1). A comparative analysis of the kinematical and dynamic transmission features of the transmission used both as speed reducer and speed increaser is presented in the paper with the aim to identify the possibilities of accomplishing the conditions of high multiplication ratios (in the range 5...30), high efficiencies and reduced overall sizes. The algorithm used to analyze and compare the values of the multiplication ratio (i) and efficiency in both cases (speed reducer (ita_r) and speed increaser (ita_i)) is also presented in the paper. The steps of the algorithm are the following: the speed reducer analysis in order to identify the transmission internal efficiency; the speed increaser analysis in order to identify the gears teeth numbers and the efficiency; the comparative analysis of the speed reducer and speed increaser.

\section{The Analysis of the Transmission as Speed Reducer}

The first step consists in the establishment of the internal transmission ratio and internal efficiency, which correspond to the reduction ratio and efficiency of the speed reducer offered by Onvio Company (Fig. 3,a).

Therefore, the speed reducer $\mathrm{M}_{04}$ was selected from the company catalogue [5], being characterized by a reduction ratio of $i=n_{i} / n_{o}=5000 / 22=227$ and an efficiency of $\eta_{r}=80 \%$. By knowing these values, the internal transmission ratio $i_{0}$ and the internal efficiency $\eta_{0}$ can be obtained according to relations (2) and (5), [2]:

$$
\begin{gathered}
i_{H 1}^{4}=\frac{\omega_{H 4}}{\omega_{14}}=\frac{\omega_{H}-\omega_{4}}{\omega_{1}-\omega_{4}}=\frac{1}{1-\frac{\omega_{1}}{\omega_{4}}}=\frac{1}{1-i_{0}}=227 \\
i_{0}=\frac{i_{H 1}^{4}-1}{i_{H 1}^{4}}=\frac{226}{227}=0,9956 \\
\eta_{r}=\eta_{H 1}^{4}=\frac{-\omega_{14} T_{1}}{\omega_{H 4} T_{H}}=\frac{-T_{1} / T_{H}}{\omega_{H} / \omega_{1}}=\frac{-\overline{i_{H 1}^{4}}}{i_{H 1}^{4}}=\frac{1-i_{0}}{1-i_{0} \eta_{0}^{w}} \\
w=\operatorname{sgn}\left(\omega_{1 H} T_{1}\right)=\operatorname{sgn}\left(\frac{\omega_{1 H} T_{1}}{-\omega_{14} T_{1}}\right)=\operatorname{sgn}\left(\frac{i_{0}}{1-i_{0}}\right)
\end{gathered}
$$

where $\overline{i_{1 H}^{4}}=\frac{T_{H}}{T_{1}}$.

For the value of the internal multiplication ratio of $i_{0}=0.9956$, it is obtained $w=+1$.

Considering relations (3) and (4), the value of the internal efficiency becomes:

$$
\eta_{0}=\frac{\eta_{H 1}^{4}+i_{0}-1}{\eta_{H 1}^{4} \cdot i_{0}}=\frac{0.8+0.9956-1}{0.8 \cdot 0.9956}=0.9989
$$

The value of the internal efficiency $\eta_{0}$ will be used as input data in the analysis of the transmission as speed increaser. 


\section{The Analysis of the Transmission as Speed Increaser}

In order to analyze the transmission as speed increaser, the value of the multiplication ratio is considered preset ( $i=i_{1 H}^{4}$ ); for this ratio, the synthesis of the teeth numbers is made and, afterwards, the speed increaser efficiency is established.

The value of the multiplication ratio is imposed according to the costumer needs and the geographical conditions in which the system is placed (wind speed or water discharge and head). Further, the algorithm is presented on the basis of an example of Onvio type transmission, characterized by a multiplication ratio of 30 .

\section{A. The Teeth Numbers Synthesis}

Considering different values of the transmission's teeth numbers, the internal transmission ratio $\left(i_{0}\right)$ and multiplication ratio $(i)$ are established; the values are presented in Fig. 4, in terms of the teeth number of gear 2 $\left(z_{2}\right)$. The diagram allows the establishment of the transmission's teeth numbers for the imposed multiplication ratio (see Fig. 3 and 4).

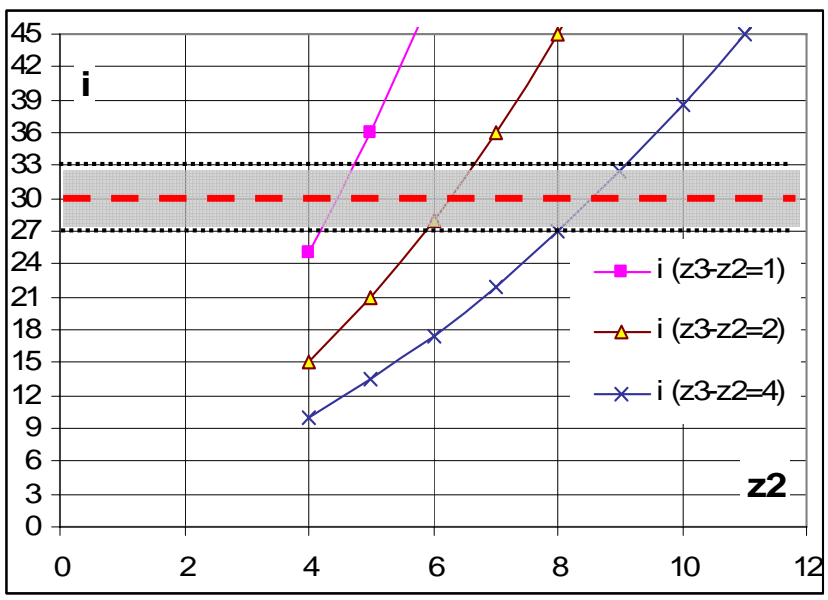

Fig. 4. Numerical simulation of the multiplication ratio for different values of the satellites' teeth numbers $z_{3}-z_{2}$ (Fig. 3)

The integer value of $z_{2}$ is found with an accuracy of $10 \%$; the other values of the teeth numbers $\left(z_{1}, z_{3}, z_{4}\right)$ are obtained in terms of $z_{2}$. From Fig. 4 can be seen that the only integer values of $z_{2}$ that fulfil the condition of a multiplication ratio of $30 \pm 10 \%$ (see the grey zone from Fig. 4) are $z_{2}=6$ for $z 3-z 2=2$ and $z_{2}=8, z_{2}=10$ for $z 3$ $\mathrm{z} 2=4$. The analysis further presented is based on an example of a transmission characterized by a difference between the teeth numbers of the sun gears and satellite gears from 1 to 4 (in order to obtain reduced overall dimensions).

\section{B. Efficiency Establishment}

The internal transmission ratio is calculated in terms of the teeth numbers, which were previously established:

$$
i_{0}=i_{14}^{H}=i_{12} \cdot i_{34}=\frac{z_{2}}{z_{1}} \cdot \frac{z_{4}}{z_{3}} .
$$

The speed increaser efficiency $\left(\eta_{i}=\eta_{1 H}^{4}\right)$ is obtained in terms of the transmission internal efficiency, given by relation (5) and the internal transmission ratio $i_{0}$, given by relation (6):

$$
\eta_{i}=\eta_{1 H}^{4}=\frac{-\omega_{H 4} T_{H}}{\omega_{14} T_{1}}=\frac{-T_{H} / T_{1}}{\omega_{1} / \omega_{H}}=\frac{-\overline{i_{1 H}^{4}}}{i_{1 H}^{4}}=\frac{1-i_{0} \eta_{0}^{X}}{1-i_{0}}
$$

where $x=-w$.

In order to analyze the transmission functioning as speed increaser, the values of the reduction / multiplication ratios $(i)$ and of the efficiencies $\left(\eta_{r} / \eta_{i}\right)$ are graphically illustrated to allow comparison (in Fig. $5-12$, the reducer efficiency is denoted $\eta_{r} \equiv i t a_{-} r$ and the increaser efficiency $\eta_{i} \equiv i t a \_i$ ).

\section{Comparative Analysis}

In order to perform the comparative analysis of the speed reducer and speed increaser features, the values of the multiplication ratio (i), speed reducer efficiency (ita_r) and speed increaser efficiency (ita_i) are illustrated in Fig. 5, 7 and 9.

The numerical simulation of the reduction and multiplication ratios is presented superposed in Fig. 5,a, $7, \mathrm{a}, 9$,a, the reducer and increaser efficiencies are presented in Fig. 5,b, 7,b, 10,b and Fig. 5,c, 7,c, 10,c, respectively.

In order to compare the chosen transmission in both cases (speed reducer and speed increaser), the multiplication ratio (at scale 1:100) and the reducer and increaser efficiencies are superposed in Fig. 6, 8 and 10, for each considered case.

The multiplication ratio and the efficiency are represented in Fig. 11 and 12 in order to analyze easily the transmission taken as speed increaser, for each case, in particular.

The simulations were made by taking into account the following premises:

1) the reducer and increaser have the same values for the internal parameters $i_{0}$ and $\eta_{0}$;

2) the value of the satellite 2 teeth number $\left(z_{2}\right)$ is imposed (see Fig. 3,b): $z_{2}=4,5,6, \ldots, 30$;

$3)$ the difference between the satellite teeth numbers is modified successively, as follows: $z_{3}-z_{2}=1, \quad z_{3}-z_{2}=2, \quad z_{3}-z_{2}=4$;

4) the difference between the teeth numbers of the sun gears and satellite gears is modified as follows: $z_{1}=z_{2}+1, z_{1}=z_{2}+2, z_{1}=z_{2}+3$;

5) the difference between the teeth numbers of sun gear 1 and satellite gear 2 is equal to the difference between the teeth numbers of sun gear 4 and satellite gear 3 . 


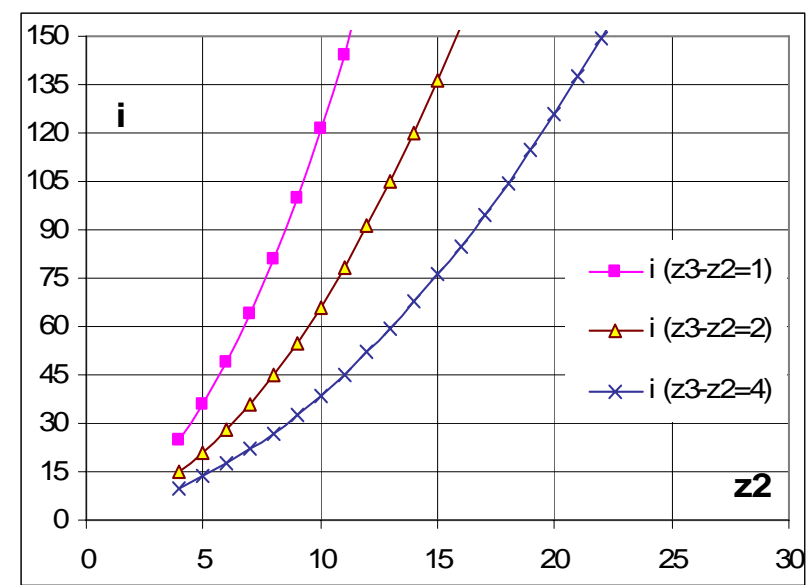

a)

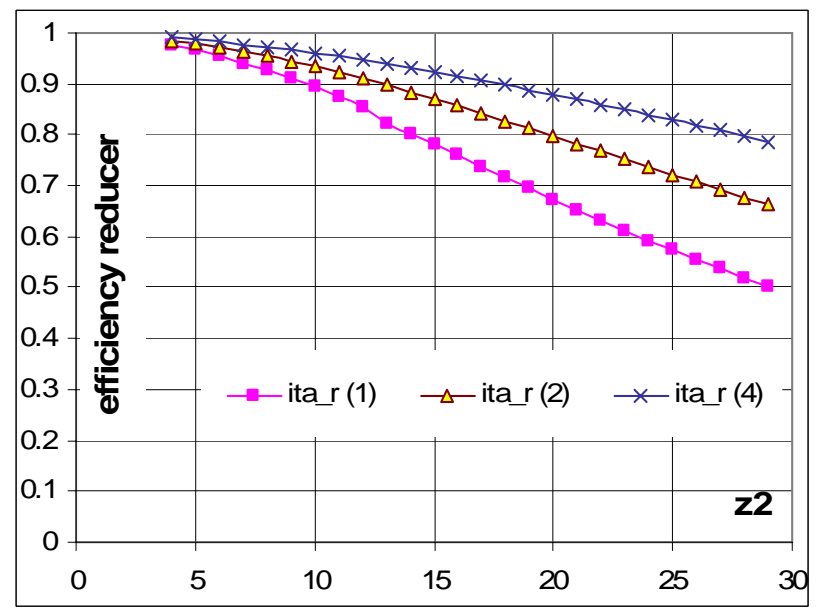

b)

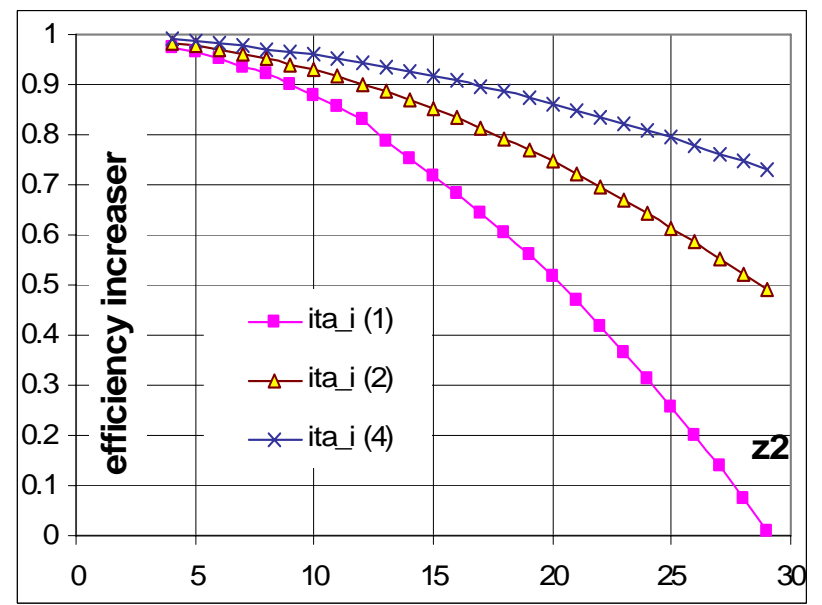

c)

Fig. 5. Numerical simulation for case $z_{1}=z_{2}+1$, of the: a) reduction and multiplication ratio, b) cycloid reducer efficiency, c) cycloid increaser efficiency

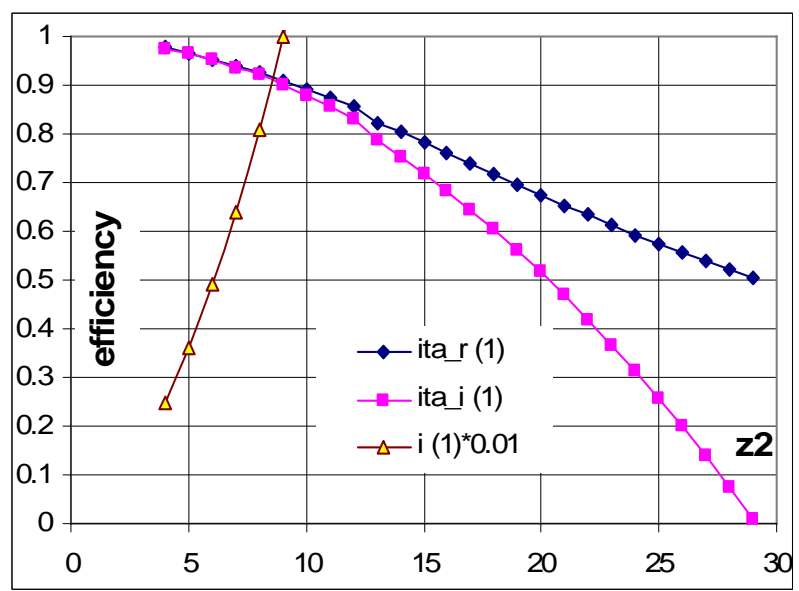

a)

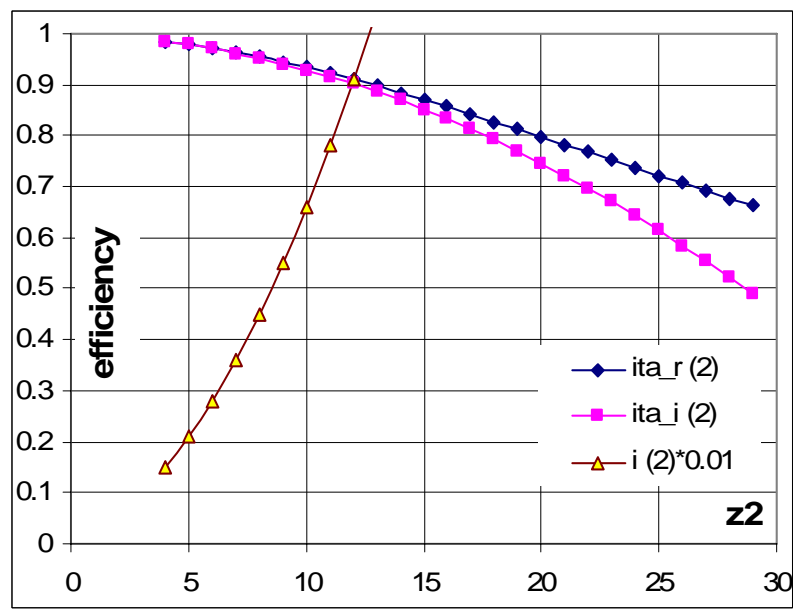

b)

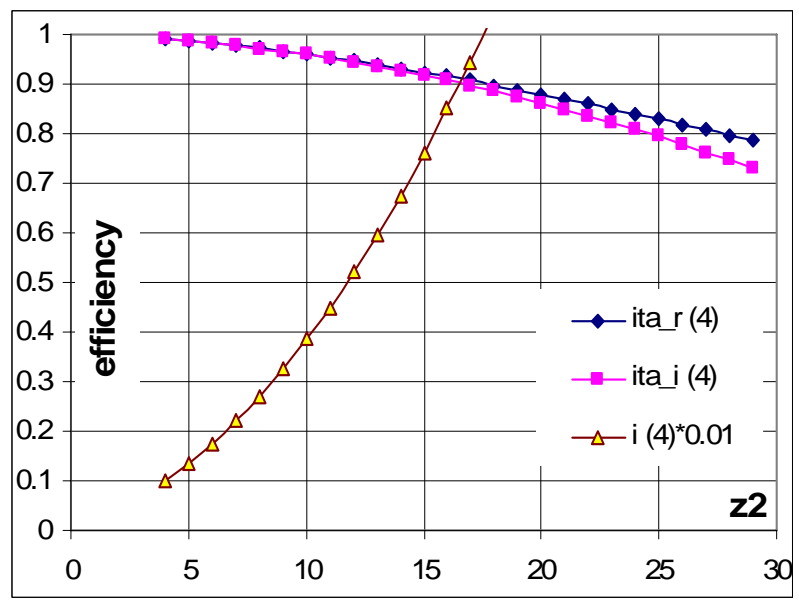

c)

Fig. 6. Numerical simulation for case in which $z_{1}=z_{2}+1$ and reduction and multiplication ratio, cycloid reducer efficiency and cycloid increaser efficiency are superposed in 3 cases: a) $z_{3}-z_{2}=1$, b) $z_{3}-z_{2}=2$ and c) $z_{3}-z_{2}=4$. 


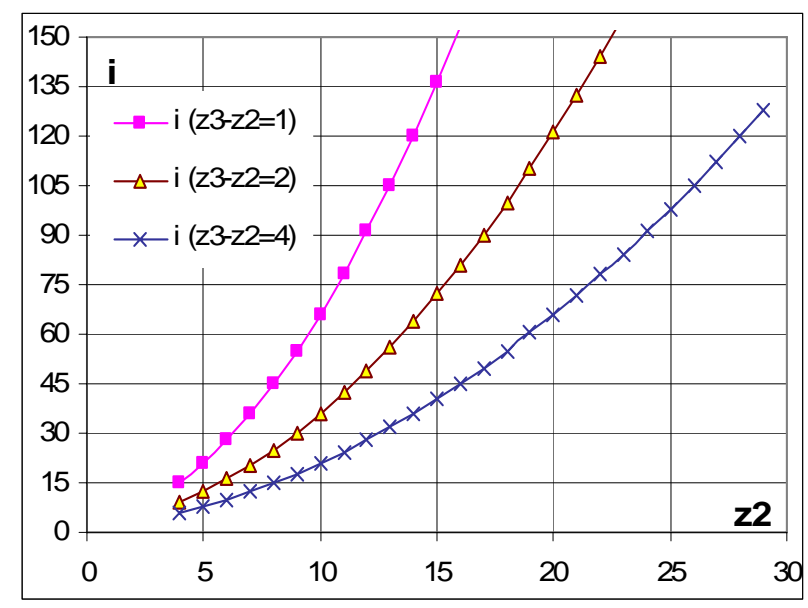

a)

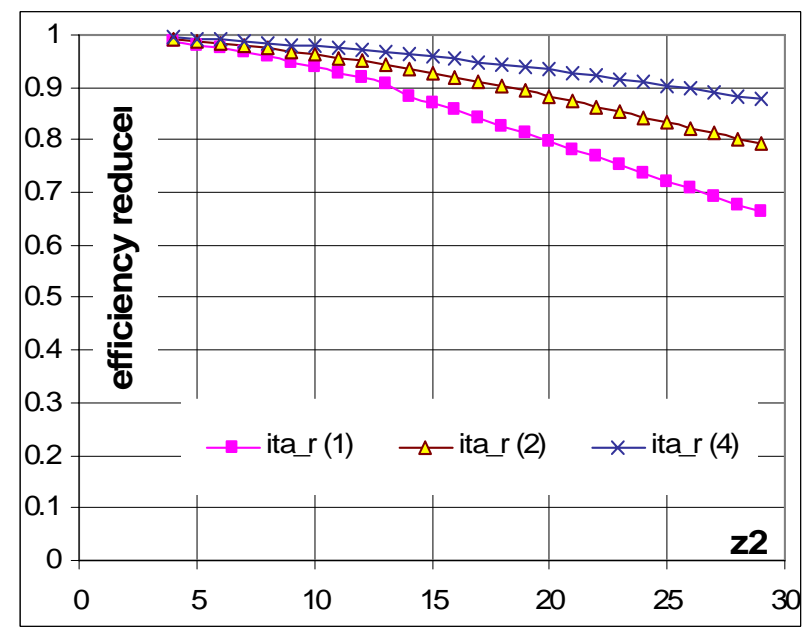

b)

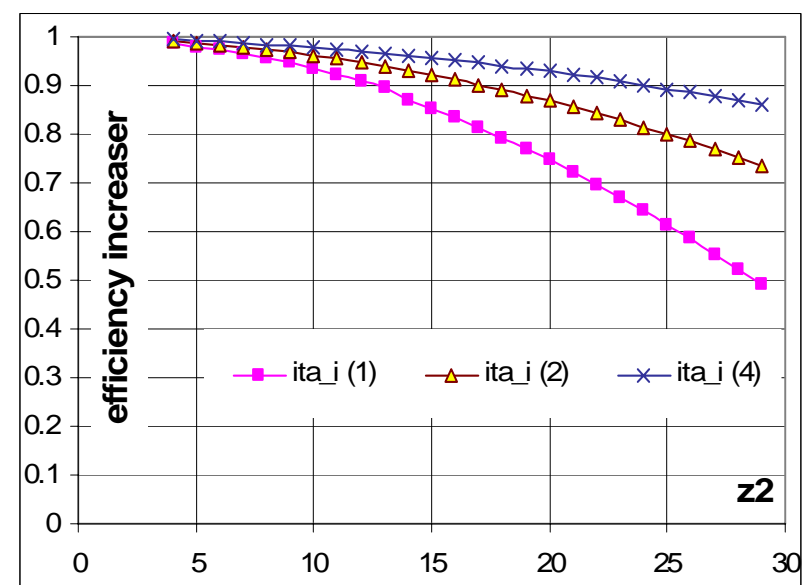

c)

Fig. 7. Numerical simulation for case $z_{1}=z_{2}+2$, of the: a) reduction and multiplication ratio, b) cycloid reducer efficiency, c) cycloid increaser efficiency

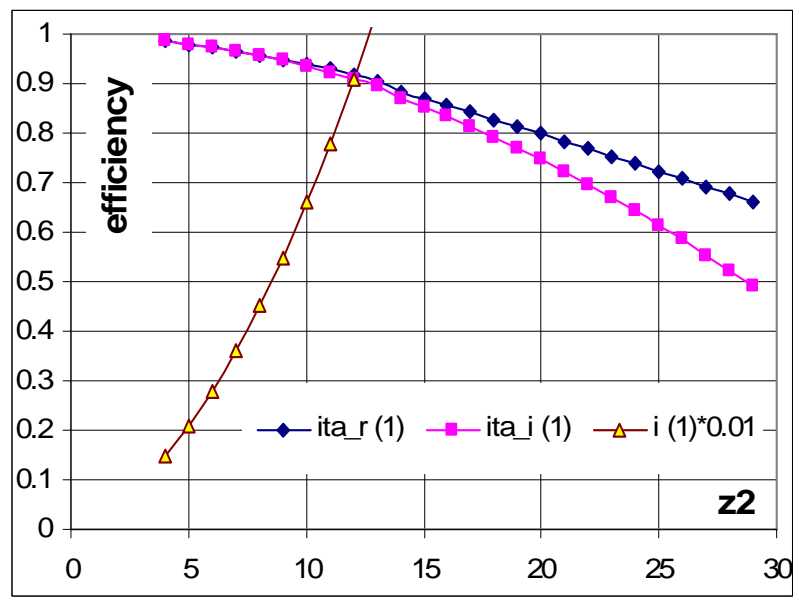

a)

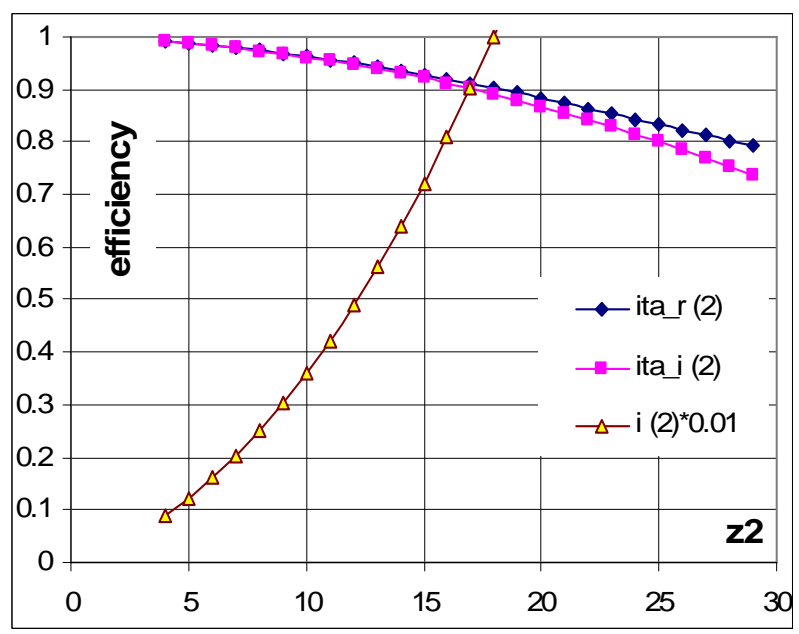

b)

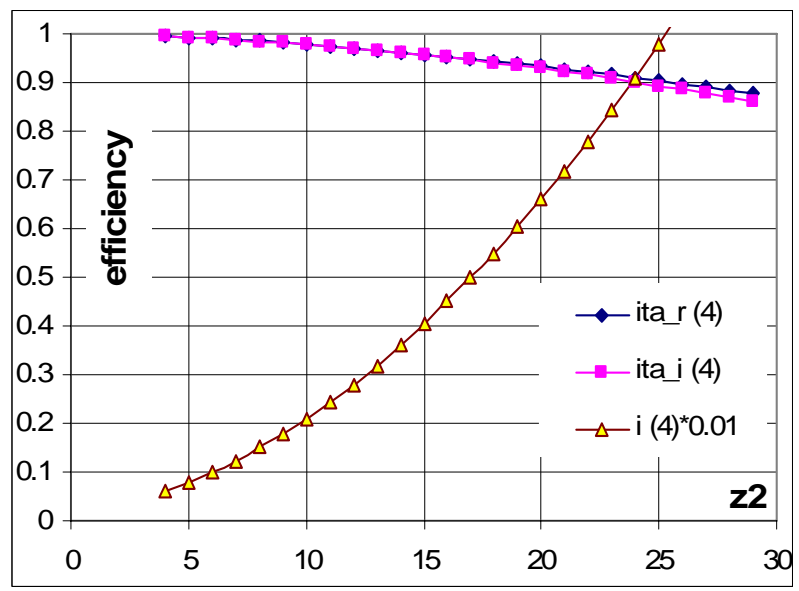

c)

Fig. 8. Numerical simulation for the case in which $z_{1}=z_{2}+2$ and the reduction / multiplication ratio, cycloid reducer efficiency and cycloid increaser efficiency are superposed, for 3 cases: a) $z_{3}-z_{2}=1$, b) $z_{3}-z_{2}=2$ and c) $z_{3}-z_{2}=4$. 


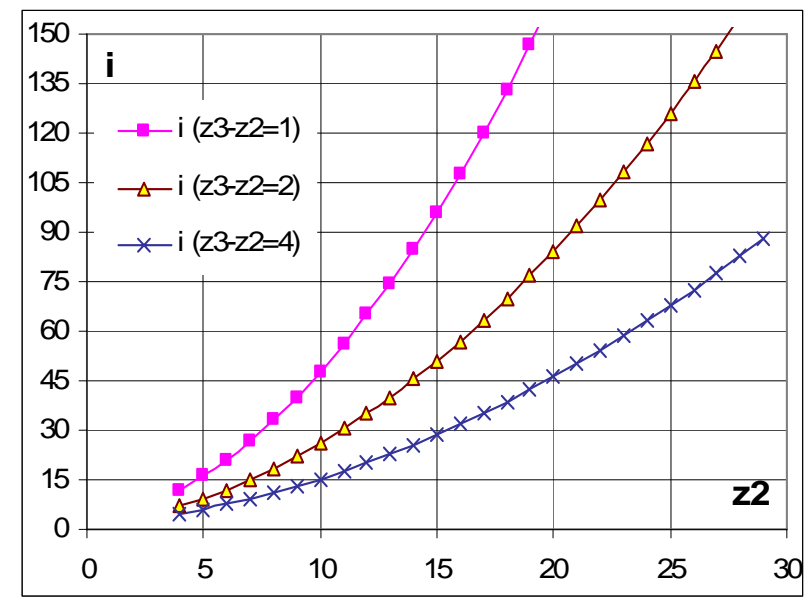

a)

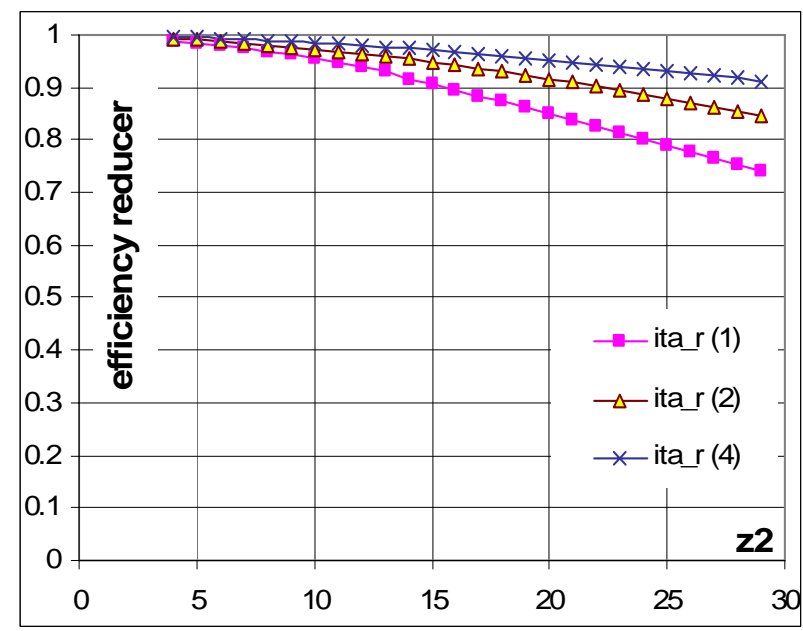

b)

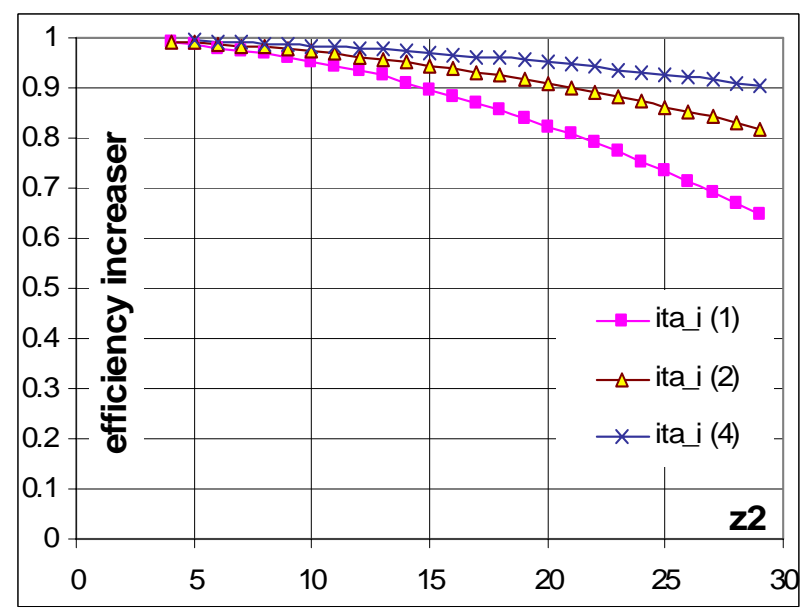

c)

Fig. 9. Numerical simulation for case $z_{1}=z_{2}+3$, of the: a) reduction and multiplication ratio, b) cycloid reducer efficiency, c) cycloid increaser efficiency

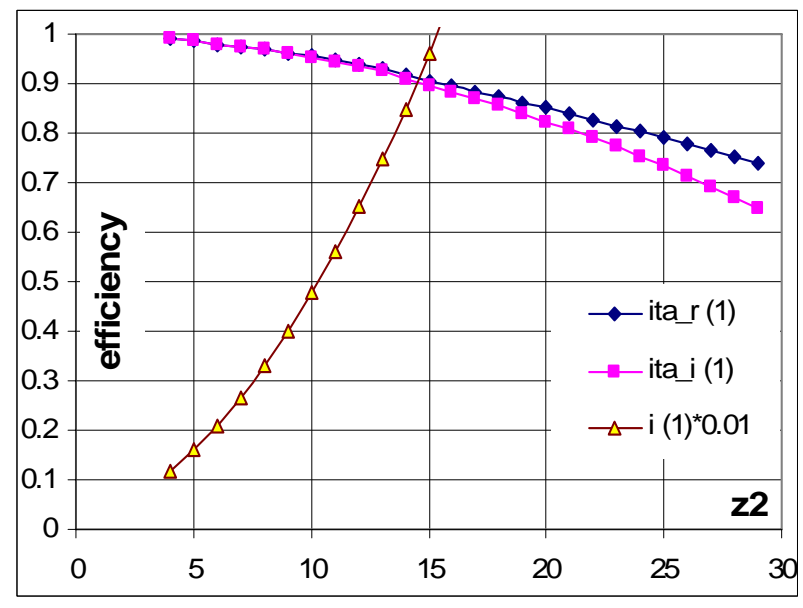

a)

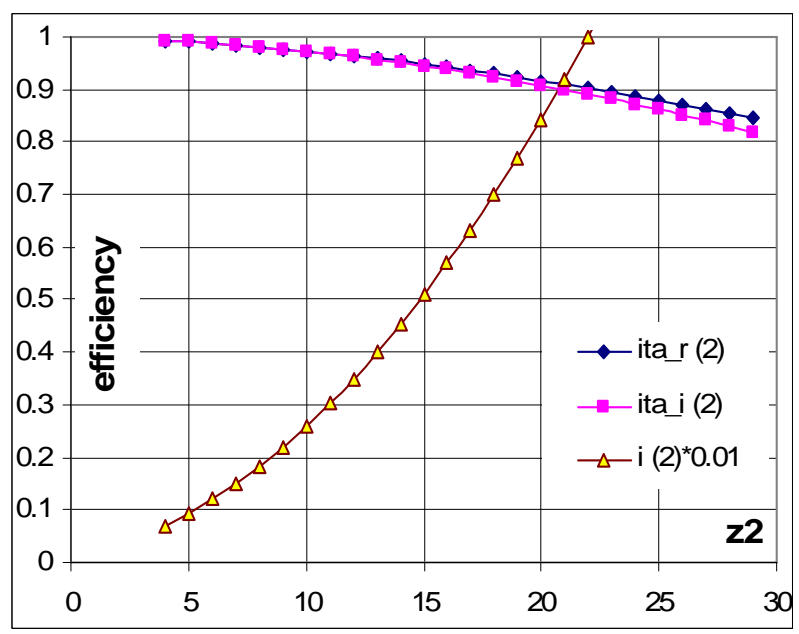

b)

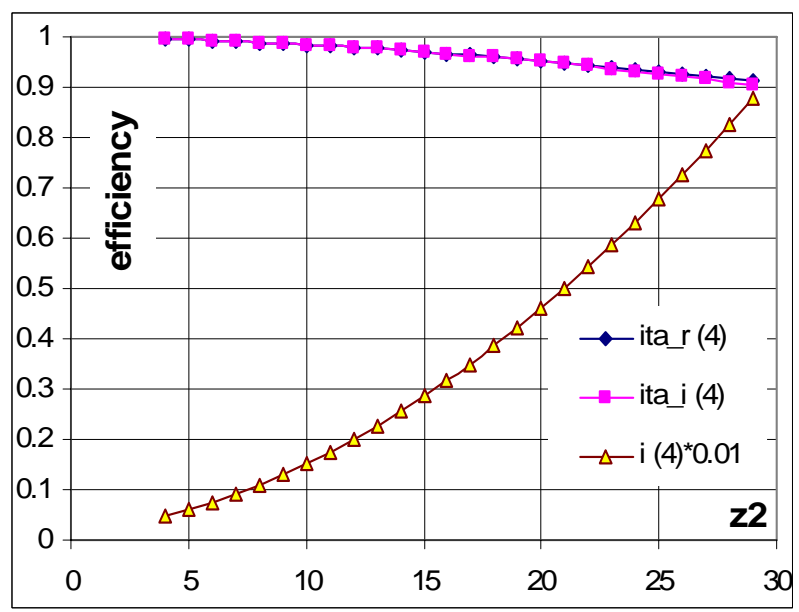

c)

Fig. 10. Numerical simulation for the case in which $z_{1}=z_{2}+3$ and the reduction / multiplication ratio, cycloid reducer efficiency and cycloid increaser efficiency are superposed, for 3 cases: a) $z_{3}-z_{2}=1$, b) $z_{3}-z_{2}=2$ and c) $z_{3}-z_{2}=4$. 


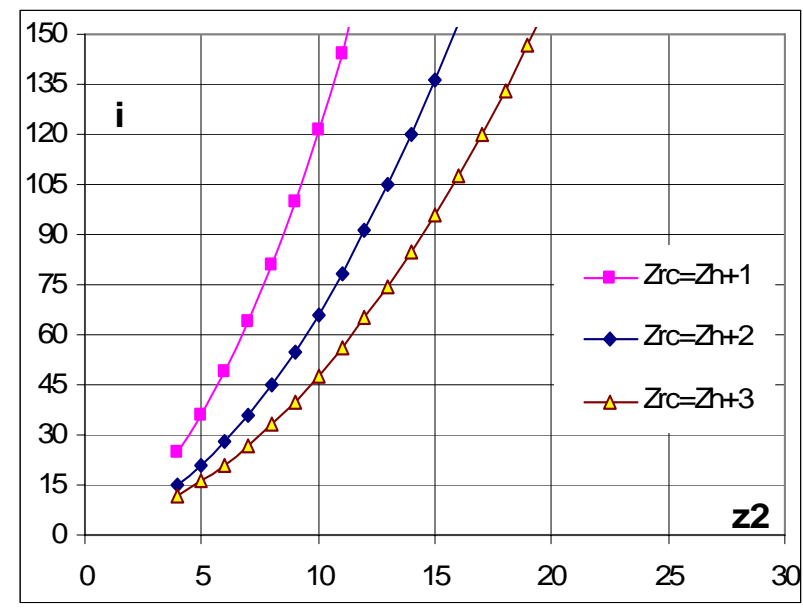

a)

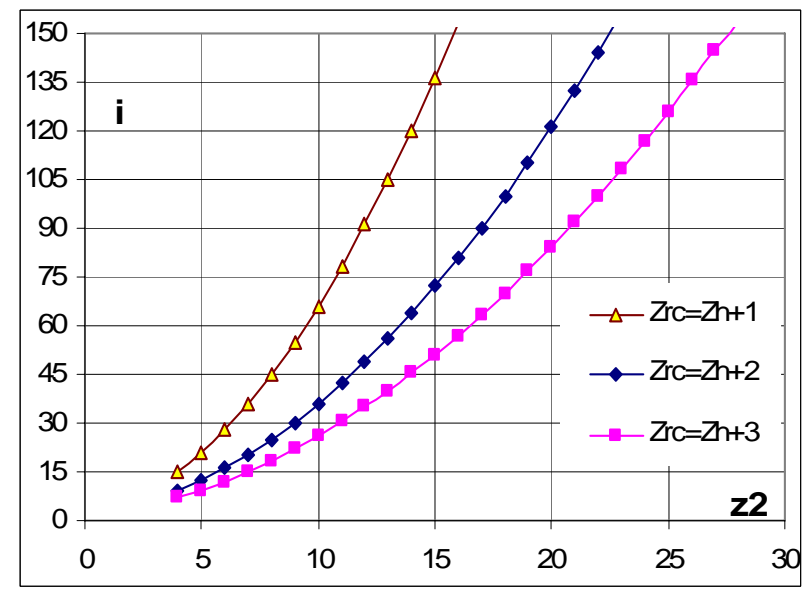

b)

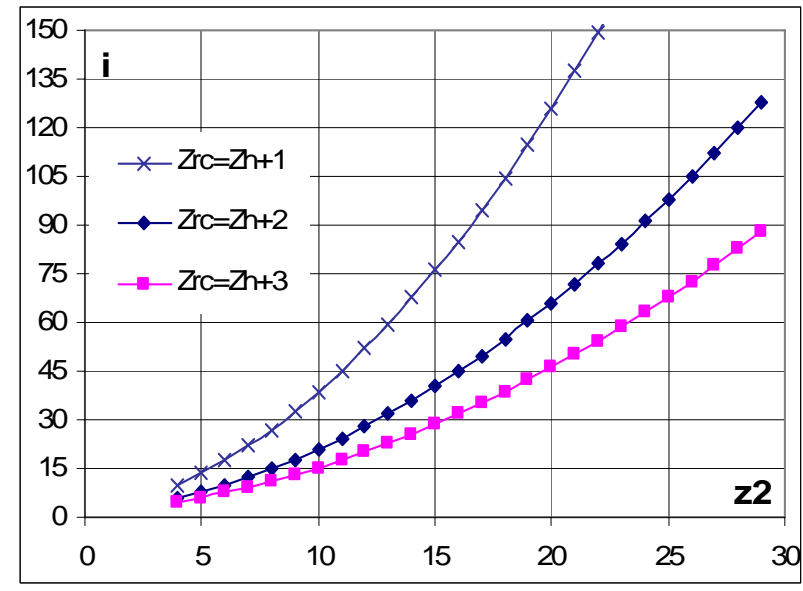

c)

Fig. 11. Numerical simulation of the multiplication ratio, for the difference between the teeth numbers of the sun gears $\left(z_{r c}\right)$ and satellite gears $\left(z_{h}\right)$, of:

a) $z_{3}-z_{2}=1$, b) $z_{3}-z_{2}=2$ and c) $z_{3}-z_{2}=4$.

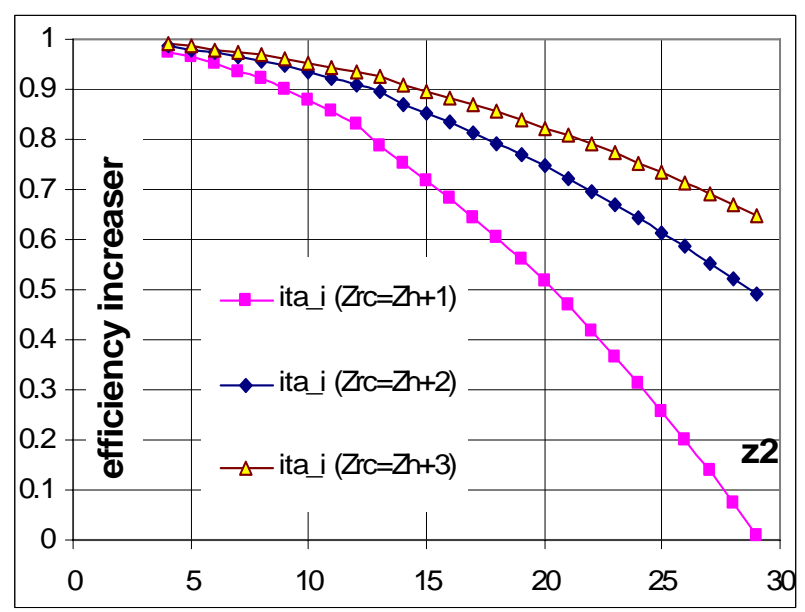

a)

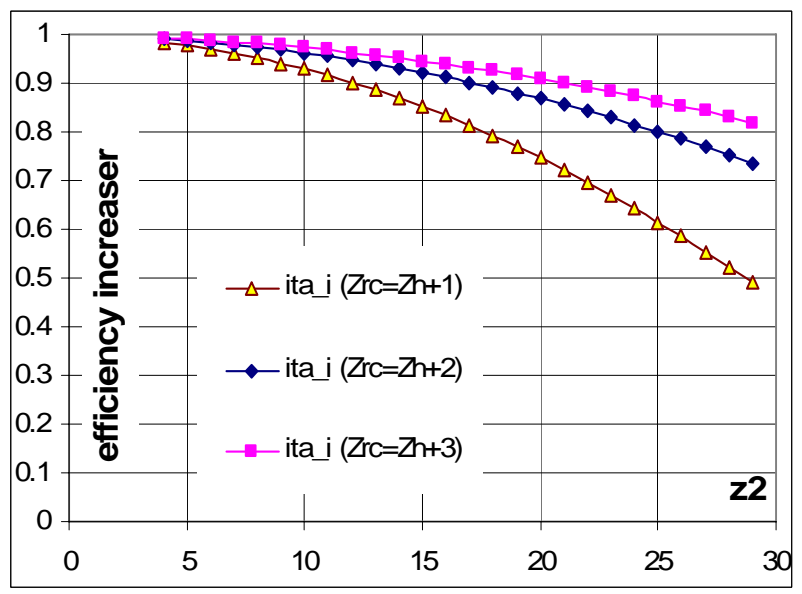

b)

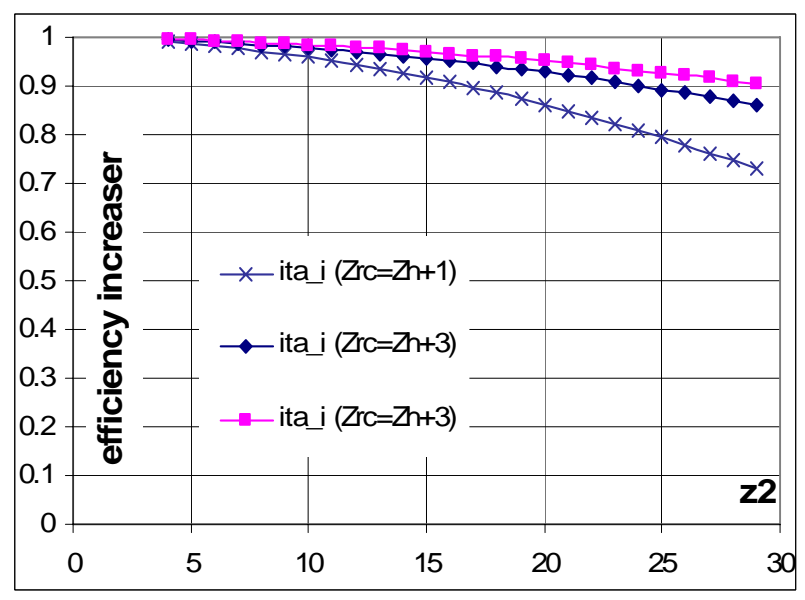

c)

Fig. 12. Numerical simulation of the increaser efficiency, for the difference between the teeth numbers of the sun gears $\left(z_{r c}\right)$ and satellite gears $\left(z_{h}\right)$, of:

a) $z_{3}-z_{2}=1$, b) $z_{3}-z_{2}=2$ and c) $z_{3}-z_{2}=4$. 


\section{Comments and Conclusions}

In order to analyze comparatively the transmission used as speed reducer and speed increaser, the following steps were considered:

1) the internal efficiency of the transmission used as reducer was established, in order to be used in the increaser analysis,

2) the synthesis of the teeth numbers for the transmission used as speed increaser was performed; the increaser efficiency was established,

3) the prerequisites for the comparative analysis of the speed reducer and increaser were established.

The results of the numerical simulations are illustrated in Fig. $5 \div 12$, which allow the following conclusions to be formulated:

1) the condition of a multiplication ratio of $30 \pm 10 \%$ (Fig. 4), characteristic for the wind plants is obtained for the following values of the teeth numbers: $z_{2}=6$ for $z_{3}-z_{2}=2$ and $z_{2}=8, z_{2}=10$ for $\mathrm{Z}_{3}-\mathrm{Z}_{2}=4$; the efficiency in these cases (see Fig. 6) is over $90 \%$;

2) for the same value of the satellite 2 teeth number $z_{2}$ and with the increase of the difference between the teeth numbers of the two satellites, the multiplication ratio decreases, the increaser efficiency increases and the difference between the reducer and increaser efficiencies decreases;

3) for the same value of $z_{2}$, and by increasing the difference between the teeth numbers of the sun gears and satellite gears, the multiplication ratio decreases, the increaser efficiency increases and the difference between the reducer and increaser efficiencies decreases;
4) for the same values of the considered parameters, the reducer efficiency is higher than the increaser efficiency;

5) by decreasing the multiplication ratio, the increaser efficiency increases, but, the transmission overall size is increasing, too;

6) the considered speed increaser fulfils the requirements for hydro stations transmission for certain values of the internal transmission ratio and efficiency, enlarging thus, the data base of speed increasers usable in hydro and wind applications.

\section{References}

[1] A. Harvey, Micro-hydro design manual, TDG Publishing, 2005.

[2] Jaliu, C., Diaconescu, D., Săulescu, R. Speed multipliers for renewable energy systems-hydro and wind. International Conference on Renewable Energies and Power Quality, ICREPQ'08, Santander, Spania, 1214.03.2008, CD Proceedings, ISBN: 978-84-611-9290-8.

[3] Jaliu, C., Diaconescu, D.V., Neagoe, M., Săulescu R. Dynamic features of speed increasers from mechatronic wind and hydro systems. Proceedings of EUCOMES 08. The Second European Conference on Mechanism Science, Casino, Italia, pp. 355-373, September 2008, Springer, ISBN 987-1-4020-8914-5.

[4] Von Schon, H.A.E.C. Hydro-Electric Practice - A Practical Manual of the Development of Water Power, its Conversion to Electric Energy, and its Distant Transmission, France Press, 2007.

[5] www.onviollc.com, Download Onvio Zero Backlash Reducer Catalogue. 